AUTHOR CORRECTION OPEN

Author Correction: Surgical Standards for Management of the Axilla in Breast Cancer Clinical Trials with Pathological Complete Response Endpoint

Judy C. Boughey ${ }^{1}$, Michael D. Alvarado ${ }^{2}$, Rachael B. Lancaster ${ }^{3}$, W. Fraser Symmans ${ }^{4}$, Rita Mukhtar ${ }^{2}$, Jasmine M. Wong ${ }^{2}$, Cheryl A. Ewing ${ }^{2}$, David A. Potter ${ }^{5}$, Todd M. Tuttle ${ }^{6}$, Tina J. Hieken ${ }^{1}$, Jodi M. Carter ${ }^{7}$, James W. Jakub ${ }^{1}$, Henry G. Kaplan ${ }^{8}$, Claire L. Buchanan ${ }^{8}$, Nora T. Jaskowiak ${ }^{9}$, Husain A. Sattar ${ }^{10}$, Jeffrey Mueller ${ }^{10}$, Rita Nanda ${ }^{11}$, Claudine J. Isaacs ${ }^{12}$, Paula R. Pohlmann ${ }^{12}$, Filipa Lynce ${ }^{12}$, Eleni A. Tousimis ${ }^{12}$, Jay C. Zeck ${ }^{12}$, M. Catherine Lee ${ }^{13}$, Julie E. Lang ${ }^{14}$, Paulette Mhawech-Fauceglia ${ }^{14}$, Roshni Rao ${ }^{15}$, Bret Taback $^{15}$, Constantine Goodellas ${ }^{16}$, Margaret Chen ${ }^{15}$, Kevin M. Kalinsky ${ }^{15}$, Hanina Hibshoosh ${ }^{15}$, Brigid Killelea ${ }^{17}$, Tara Sanft $^{17}$, Gillian L. Hirst ${ }^{2}$, Smita Asare ${ }^{18}$, Jeffrey B. Matthews ${ }^{2}$, Jane Perlmutter ${ }^{19}$, Laura J. Esserman ${ }^{2}$ and I-SPY2 Investigators ${ }^{25}$

npj Breast Cancer (2019)5:2; https://doi.org/10.1038/s41523-018-0096-0

Correction to: npj Breast Cancer 4, 26 (2018) https://doi.org/ 10.1038/s41523-018-0074-6; Article Published online 17 August 2018

In the original version of the published article, Constantine Godellas was mistakenly omitted from the Author list and Author Contributions statement. Constantine Godellas has been added as the 29th Author, and is affiliated with Loyola University Medical Center, Maywood, IL, United States. The author contributions statement has been updated as follows: Author Contributions Statement: Judy C. Boughey conducted the review of current protocols, led the effort to establish new I-SPY standards and was the principal author of the manuscript. Laura Esserman co-led the review and standardization processes. Michael D. Alvarado, Rachael B. Lancaster, Fraser Symmans, W, Rita Mukhtar, Jasmine Wong, Cheryl Ewing, David Potter, Todd Tuttle, Tina Hieken, Jodi Carter, James Jakub, Henry Kaplan, Claire Buchanan, Nora Jaskowiak, Husain Sattar, Jeffrey Mueller, Rita Nanda, Claudine Isaacs, Paula Pohlmann, Filipa Lynce, Eleni Tousimis, Jay Zeck, M. Catherine Lee, Julie Lang, Paulette Mhawech-Fauceglia, Roshni Rao, Bret Taback, Margaret Chen, Kevin Kalinsky, Hanina Hibshoosh, Brigid Killelea, Constantine Godellas, and Tara Sanft provided medical and scientific expertise/opinion towards the development of I-SPY standards. Jane Perlmutter provided the patient advocate's perspective in these discussions. All I-SPY2 trial investigators participated in the review of current standards, had the opportunity to participate in and comment on proposed standards and the final manuscript. Gill Hirst and Smita Asare provided expertise on, conducted and analyzed surveys of I-SPY trial sites. Jeffrey B. Matthews provided significant input and editing throughout the manuscript development process. All authors reviewed manuscript drafts and signed off on the final manuscript. This has been corrected in the HTML and PDF version of the article.

\section{I-SPY2 INVESTIGATORS}

A. Jo Chien ${ }^{2}$, Andres Forero-Torres ${ }^{3}$, Douglas Yee ${ }^{5}$, Erin D. Ellis ${ }^{8}$, Heather S. $\mathrm{Han}^{20}$, Janice Lu ${ }^{14}$, Anne M. Wallace ${ }^{21}$, Kathy S. Albain ${ }^{16}$, Anthony D. Elias ${ }^{22}$, Amy S. Clark ${ }^{23}$, Kathleen Kemmer ${ }^{24}$

${ }^{20}$ Department of Breast Oncology, Moffitt Cancer Center, Tampa, FL, USA; ${ }^{21}$ University of California, San Diego, La Jolla, CA, USA; ${ }^{22}$ University of Colorado Denver, Denver, CO, USA; ${ }^{23}$ University of Pennsylvania, Philadelphia, PA, USA and ${ }^{24}$ Oregon Health and Sciences, Portland, OR, USA

\begin{abstract}
(c) (i) Open Access This article is licensed under a Creative Commons Attribution 4.0 International License, which permits use, sharing, adaptation, distribution and reproduction in any medium or format, as long as you give appropriate credit to the original author(s) and the source, provide a link to the Creative Commons license, and indicate if changes were made. The images or other third party material in this article are included in the article's Creative Commons license, unless indicated otherwise in a credit line to the material. If material is not included in the article's Creative Commons license and your intended use is not permitted by statutory regulation or exceeds the permitted use, you will need to obtain permission directly from the copyright holder. To view a copy of this license, visit http://creativecommons. org/licenses/by/4.0\%.
\end{abstract}

() The Author(s) 2019

\footnotetext{
${ }^{1}$ Department of Surgery, Mayo Clinic, Rochester, MN, USA; ${ }^{2}$ UCSF Heller Diller Family Comprehensive Cancer Center, San Francisco, CA, USA; ${ }^{3}$ Department of Surgery, University of Alabama at Birmingham, Birmingham, AL, USA; ${ }^{4}$ Department of Pathology, MD Anderson Cancer Center, Houston, TX, USA; ${ }^{5}$ Department of Medicine, University of Minnesota, Minneapolis, MN, USA; ${ }^{6}$ Department of Surgery, University of Minnesota, Minneapolis, MN, USA; ${ }^{7}$ Department of Pathology, Mayo Clinic, Rochester, MN, USA; ${ }^{8}$ Swedish, Swedish Cancer Institute, Seattle, WA, USA; ${ }^{9}$ Department of Surgery, University of Chicago, Chicago, IL, USA; ${ }^{10}$ Department of Pathology, University of Chicago, Chicago, IL, USA; ${ }^{11}$ Department of Hematology and Oncology, University of Chicago, Chicago, IL, USA; ${ }^{12}$ Georgetown University Medical Center, Lombardi Cancer Center, Washington, USA; ${ }^{13}$ Moffitt Cancer Center, Tampa, FL, USA; ${ }^{14}$ University of Southern California, Norris Comprehensive Cancer Center, Los Angeles, CA, USA; ${ }^{15}$ Columbia University Medical Center, New York, NY, USA; ${ }^{16}$ Loyola University Medical Centre, Maywood, IL, USA; ${ }^{17}$ Department of Surgery and Department of Medical Oncology, Yale University, New Haven, CT, USA; ${ }^{18}$ Quantum Leap Health Care Collaborative, San Francisco, CA, USA and ${ }^{19}$ Patient Representative, Ann Arbor, Michigan, USA

${ }^{25}$ Members of I-SPY 2 Investigators are listed before references.
} 